Article

\title{
Roasting Conditions and Coffee Flavor: A Multi-Study Empirical Investigation
}

\author{
Morten Münchow ${ }^{1,2,3, * \mathbb{D}}$, Jesper Alstrup ${ }^{1}$, Ida Steen ${ }^{1,2}$ and Davide Giacalone ${ }^{4, *}$ \\ 1 CoffeeMind Aps, 2500 Valby, Denmark \\ 2 Department of Food Science, University of Copenhagen, 1958 Frederiksberg, Denmark \\ 3 Specialty Coffee Association, Chelmsford CM3 4HF, UK \\ 4 Department of Technology and Innovation, University of Southern Denmark, 5230 Odense, Denmark \\ * Correspondence: morten@coffee-mind.com (M.M.); dg@iti.sdu.dk (D.G.)
}

Received: 29 January 2020; Accepted: 20 April 2020; Published: 8 May 2020

\begin{abstract}
This research investigates the relative importance of two roasting parameters-colour (i.e., roast degree) and time- on the sensory properties of coffee. The paper draws on data from eight studies conducted using sensory descriptive analysis with trained (in six studies) or semi-trained (in two studies) assessors, focusing on a common set of attributes. The results indicated that, while both parameters significantly affected coffee flavour, colour was the stronger predictor of the two. The effects direction for both colour and time were similar and related to the rate of non-enzymatic browning, with darker roasts/longer roasting times associated with an increase in bitterness and a decrease in acidity, fruitiness, and sweetness. With respect to roasting time, we distinguished two phases, "time to first crack", corresponding to the time between the onset of roasting and the moment where the accumulated steam pressure causes the beans to crack, and "development time", corresponding to the time elapsed from the first crack to the end of the roasting process. The results clearly indicated that, under the same colour, time variation also influenced flavour, and in particular, development time, rather than time to first crack, had the largest effect on coffee flavour.
\end{abstract}

Keywords: coffee; roasting; flavour; development time; sensory science; descriptive analysis

\section{Introduction}

Coffee is one of the most important beverage commodities traded on the global market [1,2]. With increasing demand worldwide, particularly in the specialty coffee segment, scientific sensory evaluation of coffee is increasingly sought after to understand and meet consumer demands $[3,4]$. The sensory quality of coffee is a function of all the links in the coffee production chain-plant genetics, terroir, transportation, storage, roasting, grinding, brewing - which, together with social, psychological, and situational factors, determine the final consumer experience [5-11]. A clear picture of how each link, as well as the subprocesses in each link, modulates the flavour of the coffee is necessary in order to inform product development and quality control, that is to have the opportunity to create high-quality products that consistently give a satisfying consumer experience $[5,10]$.

From a sensory perspective, roasting has a major influence on the quality of coffee and is thus an important aspect for product differentiation in the marketplace. Deliberate modulation of the roasting process based on knowledge of the target consumer is therefore an important part of the product design process and quality control $(\mathrm{QC})$ protocols. This calls for reliable evidence-based knowledge of which 
input factors modulate the coffee flavour, and specifically how input (roast control parameters) and output (sensory attributes) factors are related.

The main non-volatile constituents of Arabica green beans are water $(\approx 12 \%)$, cellulose $(\approx 50 \%)$, protein $(\approx 10 \%)$, mono- and oligo-saccharides $(\approx 10 \%)$, free amino acids $(\approx 1 \%)$, chlorogenic acid $(\approx 7 \%)$, and aliphatic acids such as citric, malic, and tartaric $(\approx 2 \%)$ [12-14]. The beans also contain $\approx 15 \%$ lipids, which are not reactive during the roasting process other than being pushed to the surface during dark and/or fast roasts. During coffee roasting, the green beans undergo a transformation caused by a drastic increment in temperature from room temperature to temperatures around and often exceeding $200{ }^{\circ} \mathrm{C}$. The heat brings the green bean into pyrolysis, where volatiles and other non-volatiles are developed from the non-volatile precursors. The majority of the coffee flavour during roasting is developed via non-enzymatic browning reactions such as the Maillard reaction and caramelisation, leading to a range of coffee specific aromas such as sweet/caramel, earthy, roasted, smoky, fruity, and spicy [13,14]. The reactions also lead to non-volatile substances, of which two are the main contributors to bitterness in coffee: melanoidins from the browning reactions and the fragmentation of chlorogenic acid into quinic and caffeic acid [15]. Caffeine is another source of bitterness, but the natural content of caffeine in the green beans is hardly affected by the roasting process, due to the heat stable nature of the molecule [16]. In addition to the natural content of aliphatic acids already mentioned, carbohydrates are fragmented into smaller aliphatic acids such as formic, acetic, glycolic, and lactic acid, which contribute to acidity in different phases of the roast [17]. During the roasting process, the beans lose between 15 and 22\% of their weight, of which most is water as it ends up as a rather dry product with between 1 and $2.5 \%$ moisture [18]. From a physical perspective, it gains from around $50 \%$ to almost $100 \%$ increased volume $[13,14]$.

The application of various time-temperature relationships in the roasting process, and the following effects on chemical composition, have been investigated by several authors in the past [18-23] (see also $[7,24]$ for recent reviews). In the scientific literature, the roasting process lasts for as low as $4 \mathrm{~min}$ to almost $15 \mathrm{~min}$, depending on the roast style $[14,18,20]$. Commodity coffee is sometimes roasted in 1-3 min, which is referred to as "flash roasting" [13,25].

From a roast control perspective, most existing research has focused exclusively on overall roasting time by running isothermal high temperature short time (HTST) and low temperature long time (LTLT) roast profiles (a term indicating that the time-temperature path of the process is monitored and considered important) and related this to the resulting aroma chemistry of the coffee [18]. To the best of our knowledge, no previous research has focused on distinct sub-phases of the roasting process, and none of them evaluated sensory properties directly using sensory descriptive analysis. We recently addressed this topic in two papers where we demonstrated the important effect of roasting time and temperature on volatile formation and the sensory quality of coffee [26,27]. While these previous studies focused on common roasting-related defects, research on subtler levels of process variation is scant. Moreover, there currently seems to be a gap between the scientific literature and the growing industry trend in the last two decades where roasters, in addition to total roasting time and roast colour, have additionally placed much emphasis on the occurrence of the so-called "first crack" [28]. The first crack is an event between the onset of roasting and the termination of the roasting process where the accumulated steam pressure causes the beans to crack and expel steam along with other volatile compounds $[18,29]$. This first phase is primarily endothermic due to the heating of water, which gives a high demand for energy input in the beginning of the roasting process. Conversely, at the end of the coffee roasting process, there is a much lower need for heat input, as the process itself becomes progressively more exothermic. The added heat takes the material into a state of pyrolysis where the first important chemical reaction is the Maillard reaction in which amino acids and sugars react to form various components and particularly coffee specific aromas $[6,13]$. Thus, the time elapsed from the first crack to the end of the roasting process should be expected to be the time where the aroma formation speed is the highest [18]. 
The other major gap in the literature is that most results of roast profile modulation experiments were only reported in terms of the effects on aroma chemistry (e.g., $[17,18,20,30])$, with neither sensory descriptive analysis, nor consumer test data, save for very few exceptions [5,31]. This severely limits the usefulness of this research for the specialty coffee community: in part because it may be hard for coffee professionals (who may lack a deep background in flavour chemistry) to make practical sense of a list of reported aroma compounds and most importantly because of the inherent difficulty in reliably predicting the flavour of coffee based on its chemical make-up [5].

Clarke and Vitzthum [13] suggested a framework for reporting all relevant information in research on coffee roasting; here, the colour of the roasted coffee and total roast time were included in the necessary category of information to report, whereas "time of bean popping" (i.e., time to first crack) was included in the optional category for information to report. In the coffee industry, however, there seems to be no consensus over whether roast colour measurement is in and of itself an important indicator, or whether focus should instead be exclusively placed on the temperature evolution over time, or finally whether time of popping is particularly interesting compared to other aspects of the roasting time curve. The problem with any of these viewpoints is that in most cases, they are based on the individual opinions of professional roasters, rather than on carefully planned experiments with trained sensory panels (e.g., [32]). This could lead to commercial failures as, for instance, colour measurements are often left out of QC procedures in specialty coffee roasteries; if colour is indeed an important flavour modulator, this could result in consumer relevant, but unknown (not checked) batch-to-batch variations. The notion that colour has a larger impact on the flavour of coffee than different timing aspects is consistent with the results reported in [26], where multivariate analyses of sensory profiles consistently showed the main variation in the data to be between light and dark roast, whereas variation in timing conditions was less well explained. However, as previously mentioned, this study featured rather large variation in roasting conditions between the samples, so further research with subtler differences is needed to corroborate these initial findings.

To summarize, the overall aim of this research is to understand the relationship between the technical input parameters of coffee roasting and the resulting sensory properties of the coffee. To this end, we will present data from seven sensory profiling studies focusing on coffee roasted with different time and temperature profiles. All studies adopted the same methodological approaches and focused on the same set of sensory attributes, providing a solid basis for quantifying the magnitude of influence each roasting process control parameter (input) has on the sensory properties of the resulting coffees (output).

We will, in detail, focus on the following three objectives:

1. Compare the overall impact of roast colour modulation versus roast timing modulation on the sensory profile of coffee;

2. Assess the effect (magnitude and direction) of colour modulations on individual sensory attributes;

3. Assess the effect (magnitude and direction) of timing modulations on individual sensory attributes, with a focus on distinct phases of the roasting process.

\section{Materials and Methods}

\subsection{Overview of Studies}

An overview of the studies included in this paper is given in Table 1, including IDs used to refer to the individual studies throughout the papers. All studies were conducted using good sensory practices [33] (cf. Chapter 3, Principle of Good Practise) and focused on a common set of sensory attributes. Studies 1-6 were conducted under controlled laboratory conditions, using trained assessors. Studies 7 and 
8 were conducted at a central location test (CLT) using coffee professionals after receiving a short training session (see Section 2.3.2).

Table 1. Overview of the studies included in the paper. The third column indicates which part of the roasting process was varied in each individual study $(\mathrm{Col}=$ roast colour, 1 st crack = time to first crack, Dev $=$ development time). The sensory profiling data from Study 1 were previously published in [26].

\begin{tabular}{llll}
\hline Study ID & N Assessors & Roast Profile Modulation & Green Coffee \\
\hline 1 & 10 & Col, 1st crack, Dev & Kenyan, Ndaroini, Washed \\
2 & 10 & Col, Dev & Colombia, Horizontes, Washed \\
3 & 10 & 1st crack, Dev & Colombia, Horizontes, Washed \\
4 & 7 & Dev & Colombia, Horizontes, Washed \\
5 & 10 & 1st crack, Dev & Ethiopia, Sidamo, Washed \\
6 & 11 & Dev & Ethiopia, Sidamo, Washed \\
7 & 49 & Dev & Colombia, Horizontes, Washed \\
8 & 46 & Dev & Colombia, Horizontes, Washed \\
\hline
\end{tabular}

Table 1 reports the number of assessors per study $(\mathrm{N})$, which part of the roasting process was modulated, and the type of coffee used. To illustrate the roasting conditions varied across the eight studies, Figure 1 provides a stylized model of the roasting process in terms of time-temperature curves. The first time event is time to first crack (when a popping sound is first heard during the roast), which is followed by the so-called "development time", which denotes the phase in which the beans undergo the most significant chemical and physical changes $[18,27]$. As Figure 1 shows, the development time phase corresponds to the total duration of the roasting process minus the time to first crack. A more thorough description of the roasting conditions is provided in the next section.

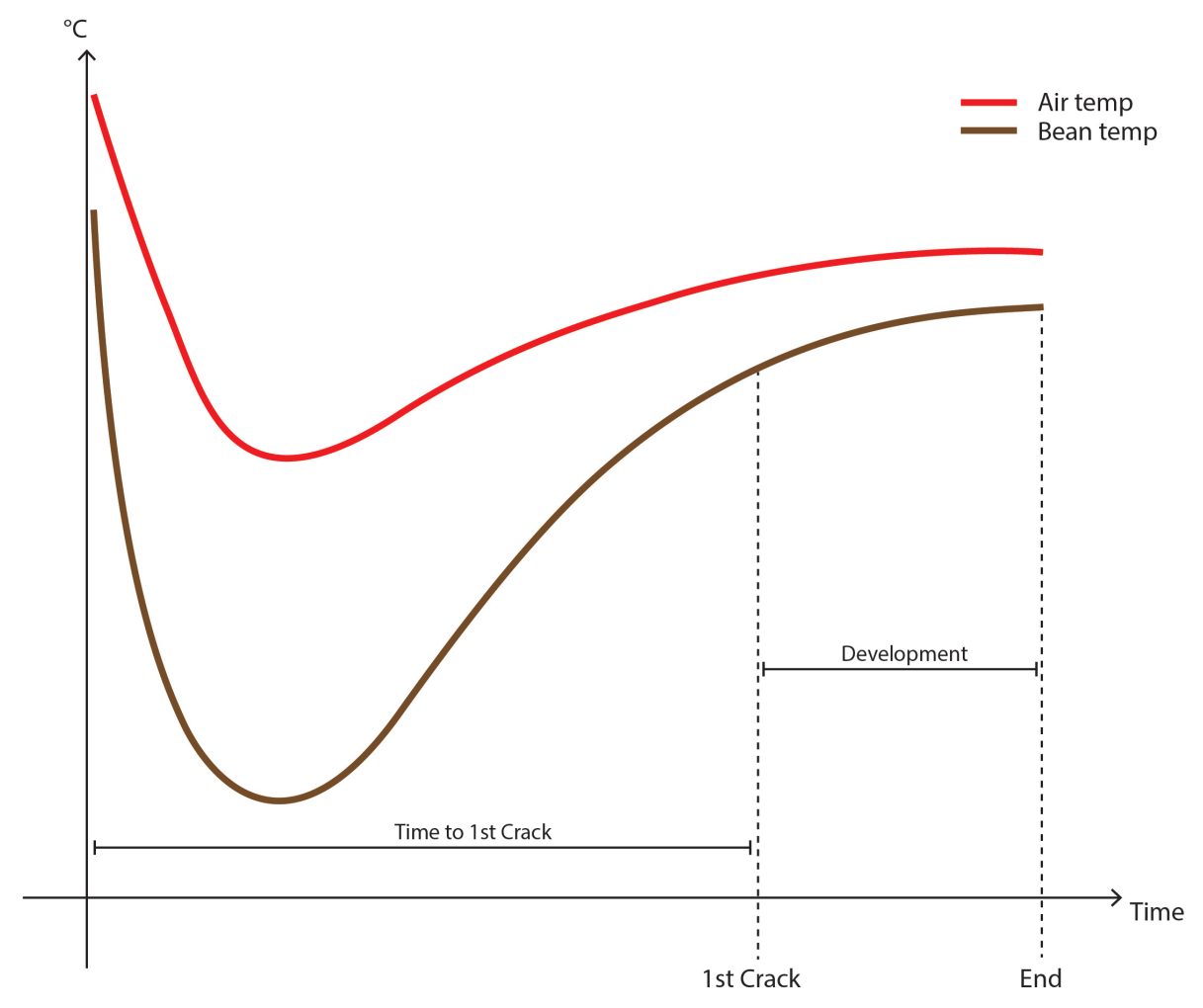

Figure 1. Model illustrating temperature development over time for the roasting profiles and the major phases (Time to first crack, development time) varied in this research. 


\subsection{Roasting Conditions}

Green beans of Arabica coffee were roasted using a Probat drum roaster (model "Probatino", $1 \mathrm{~kg}$ Probat-Werke, Dresden, Germany) in all studies, with the exception of Study 6, where an S15 Falcon (15 kg batch capacity, Loring, Santa Rosa, CA, USA) was used instead. The range of roasting conditions and, therefore, the magnitude of the modulations varied quite sizeably across studies (Table 2). In Study 1 (data previously published in [26]), major between-sample differences were introduced as that product space was intended to represent roasting related defects, and not just subtle modulations. By contrast, smaller between-sample differences in the remaining seven studies were created, based on extensive pilot work, to check whether the results would extend to subtler, but detectable differences. In order to keep consistency across studies, we chose to simplify the roasting process as much as possible, leading to the principles of high energy input in the beginning and low in the end with as few reductions as possible, and always only to reduce the speed of heat input during the roasting process.

Table 2 gives a detailed account of the roasting conditions on a sample-by-sample basis across all studies, including the two measures that will be in focus in the remainder of the paper: development time and colour of the roast. In order to keep the focus on roasting time, airflow was kept constant, and the starting temperature was kept the same within each study, except for Study 1 (Table 2). During roasting, colour was evaluated using a reference tool developed by the first author (https: / / coffee-mind.com/product/colour-palette/), which had an accuracy of \pm 1 Agtron value. After roasting, a post-hoc colour evaluation of each sample was conducted using the Javalyrics Degree of Roast Analyzer (Model JAV-RDA-D, Madison Instruments, Inc., Middleton, WI, USA) in all studies. In addition, the ColorTrack (benchtop model, FRS/ColorTrack, Inc., Santa Clara, CA, USA) was used in Studies 4-6. Each sample was measured three times, and the average value is what is reported for each sample in Table 2.

Table 2. Roasting conditions for all samples across all studies ( $\mathrm{Col}=$ Roast colour, 1 st $\mathrm{Crack}=$ time to first crack, Dev = development time), End = total roasting time. All roasting parameters are reported as time in min. The last two columns $(\mathrm{Col})$ report the Agtron Gourmet value, a spectrophotometric measure indicating the colour of the roast (with a smaller number indicating darker roasts) and the starting temperature for each sample (air temperature when the beans enter the roaster).

\begin{tabular}{ccccccc}
\hline Study ID & Sample & 1st Crack & Dev & End & Col & Start Temperature $\left({ }^{\circ} \mathbf{C}\right)$ \\
\hline 1 & 1 & $5: 41$ & $1: 50$ & $7: 40$ & 66 & 275 \\
& 2 & $10: 20$ & $6: 20$ & 18.00 & 68 & 230 \\
& 3 & $17: 58$ & $2: 30$ & $20: 20$ & 75 & 135 \\
& 4 & $9: 03$ & $2: 40$ & $11: 25$ & 74 & 210 \\
& 5 & $8: 01$ & $0: 10$ & $8: 40$ & 117 & 210 \\
2 & 6 & $9: 10$ & $4: 45$ & $13: 45$ & 46 & 220 \\
& 1 & $8: 45$ & $1: 20$ & $10: 05$ & 75 & 200 \\
& 2 & $9: 22$ & $2: 13$ & $11: 35$ & 75 & 200 \\
& 3 & $9: 20$ & $4: 12$ & $13: 32$ & 76 & 200 \\
& 4 & $9: 27$ & $5: 09$ & $14: 36$ & 76 & 200 \\
& 5 & $9: 35$ & $6: 45$ & $16: 20$ & 75 & 200 \\
& 6 & $9: 30$ & $2: 08$ & $11: 38$ & 64 & 200 \\
& 7 & $9: 15$ & $2: 20$ & $11: 35$ & 47 & 200 \\
& 8 & $9: 30$ & $2: 00$ & $11: 30$ & 90 & 200 \\
& 9 & $9: 20$ & $2: 04$ & $11: 24$ & 99 & 200 \\
\hline
\end{tabular}


Table 2. Cont.

\begin{tabular}{|c|c|c|c|c|c|c|}
\hline Study ID & Sample & 1st Crack & Dev & End & Col & Start Temperature $\left({ }^{\circ} \mathrm{C}\right)$ \\
\hline \multirow[t]{6}{*}{3} & 1 & $7: 21$ & $1: 34$ & $8: 55$ & 92 & 200 \\
\hline & 2 & $7: 58$ & 4:04 & $12: 02$ & 87 & 200 \\
\hline & 3 & $6: 56$ & 2:04 & 9:00 & 93 & 200 \\
\hline & 4 & 7:07 & $1: 49$ & $8: 56$ & 92 & 200 \\
\hline & 5 & $6: 36$ & $1: 51$ & $8: 27$ & 88 & 200 \\
\hline & 6 & $8: 37$ & $1: 23$ & $10: 00$ & 90 & 200 \\
\hline \multirow[t]{3}{*}{4} & 1 & $8: 28$ & $1: 46$ & $10: 14$ & 71 & 200 \\
\hline & 2 & $8: 33$ & $3: 12$ & $11: 45$ & 73 & 200 \\
\hline & 3 & $8: 30$ & $5: 21$ & $13: 51$ & 74 & 200 \\
\hline \multirow[t]{5}{*}{5} & 1 & $9: 17$ & $1: 30$ & $10: 47$ & 92 & 200 \\
\hline & 2 & $9: 21$ & $2: 15$ & $11: 36$ & 88 & 200 \\
\hline & 3 & $9: 17$ & $1: 50$ & $11: 07$ & 93 & 200 \\
\hline & 4 & $8: 50$ & 1:48 & $10: 38$ & 92 & 200 \\
\hline & 5 & $9: 52$ & 1:54 & $11: 46$ & 93 & 200 \\
\hline \multirow[t]{5}{*}{6} & 1 & 9:13 & $1: 23$ & $10: 36$ & 94 & 160 \\
\hline & 2 & 9:07 & 2:09 & $11: 16$ & 89 & 160 \\
\hline & 3 & $9: 14$ & $1: 48$ & $11: 02$ & 92 & 160 \\
\hline & 4 & $8: 25$ & $1: 53$ & $10: 18$ & 96 & 160 \\
\hline & 5 & $9: 25$ & $1: 55$ & $11: 20$ & 92 & 160 \\
\hline \multirow[t]{3}{*}{7} & 1 & 9:19 & $1: 56$ & $11: 15$ & 75 & 200 \\
\hline & 2 & $10: 07$ & 4:00 & $14: 07$ & 76 & 200 \\
\hline & 3 & $9: 33$ & $2: 20$ & $11: 53$ & 78 & 200 \\
\hline \multirow[t]{4}{*}{8} & 1 & $8: 52$ & $1: 31$ & $10: 22$ & 77 & 200 \\
\hline & 2 & 9:08 & $2: 23$ & $11: 31$ & 77 & 200 \\
\hline & 3 & $9: 49$ & $4: 32$ & $14: 21$ & 75 & 200 \\
\hline & 4 & $9: 31$ & $6: 30$ & $16: 01$ & 76 & 200 \\
\hline
\end{tabular}

\subsection{Sensory Descriptive Analysis}

\subsubsection{Sample Preparation}

Sample preparation was based on our previous work [26]. The coffee beans for each sample (Table 2) were ground to a slightly coarse particle size in a Mahlkönig (Hamburg, Germany) grinder. Grinding was performed within one hour prior to brewing to ensure freshness. The coffee was brewed by adding $50 \mathrm{~g}$ of coarse coffee to a French press brewer (Bodum Chambord French Press, Coffee maker) and adding $900 \mathrm{~mL}$ of hot water $\left(92^{\circ} \mathrm{C}\right)$. The mixture was gently stirred ten times with a spoon; foam was removed; and after a 3:30 min extraction time, the plunger was pressed to the bottom. The coffee was poured into thermo bottles before being poured into $200 \mathrm{~mL}$ cupping bowls and served to the sensory panel at a temperature of $55{ }^{\circ} \mathrm{C}$, deemed as optimal on the basis of previous work [34].

\subsubsection{Experimental Procedures}

Sensory evaluation of the coffee samples was conducted for each study following the principles of generic descriptive analysis [33].

The coffee samples were evaluated based on a consistent sensory vocabulary across all studies, which included basic tastes, aroma (retronasally evaluated), mouthfeel, and aftertaste attributes (Table 3). Two additional attributes of a semi-hedonic nature- "balance' and "clean cup"—-were also evaluated by the assessors, as these attributes are often included in current cupping protocols and often feature

in the sensory literature on coffee (e.g., [26,35]. All attributes were rated on a $15 \mathrm{~cm}$ line-scale with the anchor points "absent" to "a lot". The attribute list was developed by the panel for Studies 1-6, but in 
the remainder of the paper, we will focus on those attributes that were present in all studies. Studies $7-8$ used a predefined attribute list, and time was only spent on training and calibration, not on further attribute development.

The descriptive sensory analysis of these studies took place over a two-day period (one day for panel training and one day for the sensory profile assessments) and took place in individual sensory evaluation booths at either the University of Copenhagen (Studies 1-2) or at the company Kontra Coffee (Studies 3-6). The sensory panel used in Studies 1-6 consisted of trained assessors from CoffeeMind's professional sensory panel. The number of assessors varied between 7 and 11 (Table 1). By contrast, assessors in Studies 7 and 8 consisted of coffee professionals participating in the annual Nordic Roaster Forum event (www.nordicroasterforum.com). The sensory evaluation for these two studies took place in tasting facilities provided by the event organizers during the event in two consecutive years (2016, in Copenhagen, Denmark, and in 2017, in Oslo, Norway) and took place over a single day, with assessors receiving a short training and calibration session prior to the sensory evaluation focusing on attribute definition and scale usage. It should be noted, however, that while the amount of training was shorter and less extensive than in the first six studies, Studies 7 and 8 employed a much higher number of assessors (Table 1).

The assessors were instructed to evaluate the samples by the cupping method, where the coffee is aspirated into the mouth from a spoon [36]. Specifically, assessors took a $10 \mathrm{~mL}$ sample in the mouth with the spoon and swirled the coffee slowly in the mouth before spitting it out. Samples were evaluated blindly and presented to the assessors with a 3-digit code label. The coffee samples were evaluated in three replicates (four in Studies 5 and 6), and the sample order was randomized between panellists. In Studies 1 and 2, monochromatic red light was used to mask visual differences between the samples. This was not necessary in the remaining six studies as samples did not differ in colour. Water, plain toast bread, and skimmed milk were provided to the assessors to cleanse their palate between samples.

Table 3. Sensory attributes, definitions, and reference material used for assessor training across all studies.

\begin{tabular}{llll}
\hline Sensory Modality & Attribute & Definition & Reference Material \\
\hline Aroma & Roasted bread & Aroma associated with roasted bread & Roasted white toast bread \\
& Fruity & Aroma associated with a mix of fruits & $100 \%$ chocolate \\
& Cocoa & Aroma associated with cocoa beans & \\
& Nutty/chocolate & Aroma associated with nuts and chocolate & $0.6 \mathrm{~g}$ citric acid $/ \mathrm{L}$ water \\
Basic taste & Acidity & Sour taste associated with citric acid solution & $0.54 \mathrm{~g}$ caffeine $/ \mathrm{L}$ water \\
& Bitterness & Bitter taste associated with caffeine solution & $24 \mathrm{~g}$ sucrose $/ \mathrm{L}$ water \\
& Sweetness & Sweet taste associated with sucrose solution & Coffee with milk (Studies $1,3-7)$ \\
Mouthfeel & Body & Fullness of the coffee in the mouth & Xanthan gum in water $(0.07,0.1$ and $0.02 \mathrm{~g} / \mathrm{L})($ Studies 2 and 8$)$ \\
Aftertaste & Aftertaste & The length of lingering flavour after spitting out the sample & \\
Other & Balance & How well the flavours are balanced & \\
& Clean cup & No interfering negative impressions; no non-coffee like tastes or aromas & \\
\hline
\end{tabular}

\subsection{Data Analysis}

The data analytical strategy was informed by the research aims (§1.3). Pearson's correlation analysis was used to uncover the relationship between the two main roasting parameters (colour and time) and all sensory attributes. Amongst the eight datasets included in this paper (Table 1), only Study 1 and Study 2 contained colour and timing modulation in the same study, so this part of the analysis used data from these two studies only.

The second set of the analyses pertained to the sensory modulation of the coffee in the two different phases of roasting, namely time to first crack and development time, when keeping roast colour constant. To address this issue, we used a linear regression model, drawing on data from all studies, to assess the effect of the two roasting phases (time to first crack and development time) on the intensity of each sensory attribute. Upon discussion amongst the authors, it was decided not to include Study 1 in this analysis as the input parameters were rather different from those in the other seven studies (see Table 2). 
Thus, with respect to time to first crack, the value range in the final model went from a minimum of 7:21 min (Sample 1, Study 3) to a maximum of 10:07 min (Sample 2, Study 7); for development time, the minimum value was 1:20 min (Sample 1, Study 2) and the maximum 6:45 min (Sample 5, Study 2).

All analyses were conducted in the statistical environment $\mathrm{R}[37]$ using a 5\% significance level.

\section{Results and Discussion}

\subsection{Relative Impact of Roast Colour and Timing on Coffee Flavour}

First, we compared the effect of roast colour versus overall timing of the roast and then moved on to focusing on the more subtle timing differences between the first part of the roast versus end of roast.

Table 4 reports correlation coefficients between sensory attributes and timing and colour input parameters for both studies. Note that the correlation for time to first crack and end time are not given for Study 2 because in this study, the focus was on colour and development time with no variation in time to first crack (Table 4). It is easy to gauge that correlation coefficients for colour were generally larger than those for development time, as well as when considering the sensory profile of the coffee as a whole (the column "average" in Table 4). It is especially interesting to note that the total roast time in and of itself was not correlated with any flavour attributes, supporting the claim that the time coffee spends in the darker brown part of the roasting process is more important than the total time it spends in the process. In both studies, colour was primarily associated positively with bitterness, whereas it was negatively correlated with most other attributes. The size of the correlation coefficients was slightly different across the two studies, though the direction of the effects was identical. The only exception to this was the attribute "roasted bread", which was negatively correlated with colour in Study 1 and positively in Study 2.

Table 4. Correlation coefficients between roasting parameters $(\mathrm{Col}=$ roast colour, 1 st Crack $=$ time to first crack, End $=$ total roasting time, Dev $=$ development time) and sensory attributes for Studies 1 and 2. The two-tailed $p$-value associated with regression coefficient is also indicated $\left({ }^{n . s .} p>0.05 ;{ }^{*} p<0.05 ;{ }^{* *} p<0.01 ;{ }^{* * *} p<0.001\right)$. To ease interpretation, the sign of the correlation coefficient for the colour parameter was reversed as lower Agtron values indicate a darker roast. The last column includes the normalized average correlation coefficient (mean of the absolute values of each row).

\begin{tabular}{|c|c|c|c|c|c|c|c|}
\hline \multicolumn{8}{|l|}{ Study 1} \\
\hline$r_{(160)}$ & Acidity & Bitterness & Sweetness & Body & Roasted bread & Fruity & Average \\
\hline Col & $-0.18^{*}$ & $+0.66^{* * *}$ & $-0.34^{* * *}$ & - & $-0.30^{* * *}$ & $-0.57^{* * *}$ & $0.40^{* * *}$ \\
\hline Dev & $-0.18^{*}$ & $+0.42^{* * *}$ & $-0.23^{* *}$ & - & $-0.16^{*}$ & $-0.38^{* * *}$ & $0.21^{* *}$ \\
\hline 1st Crack & $+0.13^{n . s .}$ & $-0.11^{n . s .}$ & $+0.09^{n . s .}$ & - & $+0.01^{\text {n.s. }}$ & $+0.06^{n . s .}$ & $0.09^{\text {n.s. }}$ \\
\hline End & $+0.03^{n . s .}$ & $+0.10^{n . s .}$ & $-0.02^{n . s .}$ & - & $-0.06^{n . s .}$ & $-0.12^{n . s .}$ & $0.07^{n . s .}$ \\
\hline \multicolumn{8}{|l|}{ Study 2} \\
\hline$r_{(338)}$ & Acidity & Bitterness & Sweetness & Body & Roasted bread & Fruity & Average \\
\hline Col & $-0.49^{* * *}$ & $+0.59^{* * *}$ & $-0.44^{* * *}$ & $+0.13^{*}$ & $+0.63^{* * *}$ & $-0.49^{* * *}$ & $0.46^{* * *}$ \\
\hline Dev & $-0.10^{n . s .}$ & $+0.06^{n . s .}$ & $+0.08^{n . s .}$ & $-0.01^{\text {n.s. }}$ & $+0.13^{*}$ & $-0.03^{n . s .}$ & $0.07^{n . s .}$ \\
\hline
\end{tabular}

Regarding development time, the direction of the correlations with the sensory attributes was identical to that of colour, but the magnitude was lower, with many of the correlations in Study 2 failing to reach statistical significance. Within timing, however, it is relevant to note from Study 1 that development time was much more impactful than both time to first crack and end time, with correlations for these two parameters being largely non-significant (Table 4). 
Taken collectively, the results indicated that even though variation in the timing aspects of the roasting process seemed to affect the sensory properties, the colour of the roast was a stronger predictor of the flavour of coffee. With respect to the correlation observed in Study 2, an interesting observation from additional analyses (not shown here) was that assessor performance was better for samples that varied in both colour and development time (Samples 1-5) than for samples that only varied with respect to the latter (Samples 6-10). Specifically, assessor repeatability and discrimination were much higher ${ }^{1}$, suggesting that describing differences between samples when colour differences were excluded was significantly more difficult.

With respect to the direction of the correlations, colour (i.e., a darker roast) was positively correlated with bitterness and negatively correlated with sweetness, acidity, and fruitiness (Table 4). This is not surprising as it is essentially related to the further progression of the Maillard phase, with a darker roast having a higher content of dark and bitter tasting melanoidins, as well as bitter fragments of chlorogenic acids, and a lower content of sugars and chlorogenic acids as reported in the literature (e.g., [20]). The attribute "roasted bread" was positively correlated with colour (as would be expected) only in Study 2, whereas in Study 1, it was negatively associated with colour (Table 4); one should keep in mind, however, that the range of colour differences in Study 1 was however much larger than in the other studies (Table 2).

Overall, these results were very consistent with the results presented in the literature reporting that the degree of roast (i.e., colour) was associated with an increase in the intensity of bitterness and roasted attributes and a decrease in sweetness $[5,31,38]$. The observed decrease in acidity intensity was consistent with results reported for Robusta coffee in [38], whereas [31] reported no effect of roasting on this attribute.

The sign of the correlations for colour and roasting time was similar, though as already mentioned, colour was more strongly correlated with sensory variation. We look more closely at the effect of roasting time in the next section.

\subsection{Impact of Timing Variation: Which Roasting Phase Is Most Important?}

Table 5 shows the results for linear regression analyses estimating the effects of the two roasting phases on all sensory attributes across all studies (except Study 1, which, as previously explained, was excluded due to having a very different value range compared to all other studies).

The first thing to notice from Table 5 is that development time had a significant effect on all sensory attributes, whereas time to first crack only significantly affected five out of the nine attributes. Furthermore, the level of significance for development time was generally much lower $(p<0.001$ for seven out of nine attributes for development time vs. only three for time to first crack). With respect to our last aim, therefore, the results clearly showed that out of the two roasting phases, development time had a larger influence on coffee flavour than time to first crack.

With respect to the direction of the effect (possibly the most interesting aspect from an industry perspective), it is first interesting to notice the effect roasting had on the basic tastes. The regression slopes reported in Table 5 represent the rate of change of attribute intensity (in $\mathrm{cm}$ ) as a function of roasting time (in seconds). There was a negative effect of both time to first crack and development time on "acidity". Further, there was also a consistent increase in "bitterness" associated with both roasting phases. Finally, there was a significant negative effect of development time on sweetness, whereas the effect of time to first crack in this case was not significant (Table 5).

1 When considering significance $(p)$ and mean squared error (MSE) values across the panel and attributes in the PanelCheck software, the values (averaged across all attributes) in Samples 1-5 were $p=0.14$ and MSE $=6.99$, whereas for Samples 6-10, the values were $p=0.47$ and $M S E=11.23$. 
By contrast, the mouthfeel attribute "body" was not affected at all by either roasting phases, which is interesting as this goes against popular beliefs linking the Maillard reaction with texture. The alleged mechanism would be a linkage between molecular weight and viscosity, by which a higher content of melanoidins as a result of the Maillard reaction would result in higher overall molecular weight, and therefore greater viscosity and mouthfeel thickness (e.g., [10]). Nevertheless, we found no evidence supporting this link, consistent with earlier results reported in the literature [31,38]. We could therefore conclude with greater confidence that roasting had no effect on "body", especially due to the fact that our regression analysis was very highly powered ${ }^{2}$ and also the fact that at least two studies ( 2 and 8 ) were run with specific focus on mapping mouthfeel modulations as a function of roasting parameters, so the panel leader focused much of the training time especially on panel calibration for the attribute "body".

Another somewhat surprising result was that, unlike bitterness, aftertaste intensity decreased with longer roasting time in both phases (Table 5), as most of the lingering aftertaste of coffee is due to heavy and low water soluble bitter melanoidins. A possible explanation for this result is that in coffee lower in bitter and overall intensity, assessors became more perceptually aware of aftertaste notes.

The effect of roasting time on the remaining flavour attributes was less surprising; in particular, "fruitiness" was found to decrease with both roasting phases, similar to acidity. Higher intensity in "roasted bread" was significantly associated with longer development time. This was in line with the results presented in [26,27] showing the formation, during development time, of compounds such as maltol (caramel-like), difurfuryl ether (roasted), and pyridine (roasted, burnt), which are associated with this particular attribute. Following [26], the Specialty Coffee Association certification system for roasters (https: / sca.coffee/education/programs / coffee-skills-program) includes a defect called "baked" correlated with an overly extended development time and characterized by very intense roasted bread notes. The results of the regression analysis let us conclude with greater confidence that it was indeed development time, and not time to first crack, that was associated with this flavour attribute.

Finally, roasting time was negatively associated both with respect to "balance" and "clean cup", although for "balance", only the effect of development time was significant. Results for these two attributes should be interpreted with caution in light of their semi-hedonic nature; in particular, because expert scores may not necessarily correlate with consumer preferences [8] and also because in-depth analyses revealed a poor agreement between experts for these attributes [39].

Table 5. Linear regression slopes quantifying the change in sensory attributes as a function of change in timing parameters "time to first crack" and "development" time. The significance level associated with the regression coefficient is also reported $\left({ }^{n . s .} p>0.05 ;^{*} p<0.05 ;{ }^{* *} p<0.01 ;{ }^{* * *} p<0.001\right)$.

\begin{tabular}{lllll}
\hline Attribute & \multicolumn{2}{l}{ Time to 1st Crack } & \multicolumn{3}{l}{ Development Time } \\
& Slope & $p$ & Slope & $p$ \\
\hline Acidity & -0.007 & $* * *$ & -0.011 & $* * *$ \\
Bitterness & +0.005 & $*$ & +0.003 & $* * *$ \\
Sweetness & +0.000 & $n . s$. & -0.002 & $* * *$ \\
Body & +0.000 & $n . s$. & -0.000 & $* * *$ \\
Balance & -0.003 & $n . s$. & -0.005 & $* *$ \\
Clean Cup & -0.007 & $* * *$ & -0.009 & $* * *$ \\
Roasted bread & +0.003 & $n . s$. & +0.005 & $* * *$ \\
Fruitiness & -0.005 & $* *$ & -0.005 & $* * *$ \\
Aftertaste & -0.010 & $* * *$ & -0.003 & $* *$ \\
\hline
\end{tabular}

2 Given the number of observations $(N=1918)$, the chosen level of $\alpha(5 \%)$, and the observed standard deviation for this attribute (3.32), the probability of detecting a $0.5 \mathrm{~cm}$ change was over $99 \%$. 


\subsection{Limitations and Future Research}

It is important to stress that the findings reported so far apply within the conditions tested. In particular, we draw attention to the following aspects: first, all studies used washed coffee beans using either a Probat flame-on-drum roaster or a Loring convection-based roaster, so caution must be exerted when generalizing these results to other processing types (e.g., natural or pulped natural coffee) and to other roasters.

Secondly, caution should be exerted when interpreting the results in Table 5, by keeping in mind the distinction between statistical and practical significance. Taking development time as an example, the slope for "acidity" tells us that each second the bean spent in that roasting phase corresponded to a decrease in that attribute of $11 \times 10^{-4} \mathrm{~cm}$ per second. Considering a normal development time, say $3 \mathrm{~min}$, this would result in a decrease in acidity of approximately $2 \mathrm{~cm}$ on a $10 \mathrm{~cm}$ line scale, which would clearly be important in terms of characterizing different coffees. By contrast, the slopes for attributes such as sweetness and body, although significant due to the very large sample size, were very close to zero; such effects were therefore not as consequential and were more likely to be spurious.

Thirdly, the range of variation in roast colour (46-99 Agtron, with a mean value of 79) was considered the best compromise between the practises of the commodity coffee industry segment, which tends to roast very dark coffee, and the specialty coffee segment, although in reality where very light roasts (often all the way up to 120 Agtron) are commonplace. The samples used in all studies, thus, represent a roast degree that would be amongst the lightest in the industry and amongst the darkest found in the specialty segment. While this choice was motivated by making the results useful to coffee professionals from both ends of the spectrum, clearly, future research should assess whether similar findings would be obtained with a sample space that extends more in the light or dark direction.

Fourthly, the sensory descriptors included in the study were relatively generic, rather than highly specific, as for some of the studies (Studies 7 and 8 especially), there was no time to train the assessors extensively. Future extension of this line of work may go beyond basic tastes and generic flavour categories by exploring individual aspects (such as "fruitiness" or "body") more in depth.

Finally, in order to focus on the two roasting conditions, it was necessary to standardize certain experimental conditions in all studies, such as the serving temperature, the method of roasting, and the method brewing method, which are known to affect coffee flavour independently $[11,34,38,40]$. Therefore, future studies are advised to assess whether the findings replicate across other different experimental conditions, as well as the relative contribution of roasting time and colour relative to these other variables .

\section{Conclusions}

The overall aim of this research was to assess the relative importance of two roasting parameters — colour and time—on the sensory properties of coffee. Drawing on data from eight studies, the results clearly indicated that, while both parameters were significantly related to coffee flavour, colour was the stronger predictor of the two. The direction of the effects for both colour and time were similar, with darker roasts/longer roasting times associated with an increase in bitterness and a decrease in acidity, fruitiness, and sweetness. An interesting finding was that variation in roasting timing, keeping colour constant, had a systematic effect on flavour. Here, we distinguished two phases, "time to first crack", corresponding to the time between the onset of roasting and the moment where the accumulated steam pressure causes the beans to crack, and "development time", corresponding to the time elapsed from the first crack to the end of the roasting process. The latter was expected to be the primary aroma development phase, and accordingly, the results indicated that development time had a larger impact on coffee flavour than time to first crack. 
This research represents a first attempt to evaluate the effects of roasting parameters using sensory descriptive analysis with trained assessors, as opposed to "cupping" evaluation from coffee experts, which from a sensory science perspective, may be seen as unreliable due to the very small sample size, lack of sufficient calibration, the lack of replicates, etc. Future research on a wider range of roasting conditions is advised to gain a fuller picture of how roasting modulates coffee flavour. From a practical perspective, this research should provide an initial framework for the coffee industry to understand how the roast degree (i.e., colour) and roasting time affect coffee flavour. In particular, our results can be used, e.g., in evidence-based certification systems and to aid coffee roasters in their product development projects.

Author Contributions: Conceptualization, M.M. and D.G.; methodology, M.M., J.A., and I.S.; software, D.G.; formal analysis, D.G.; investigation, M.M., J.A. and I.S.; resources, M.M.; data curation, M.M. and D.G.; writing, original draft preparation, M.M. and D.G.; writing, review and editing, D.G.; visualization, M.M. and D.G.; project administration, M.M.; funding acquisition, M.M. All authors read and agreed to the published version of the manuscript.

Funding: This research received no external funding.

Acknowledgments: We thank Rob Hoos and Alexander Phul for help with the preparation and execution of Studies 3 , 5 , and 6. Rob Hoos is furthermore thanked for ongoing inspiration in the early phase of the work that led to numerous improvements of this paper. We thank Tim Wendelboe and Jens Nørgaard for working with us at Nordic Roaster Forum 2016 and 2017 (Studies 7 and 8) by allowing us access to utilizing the audience as a sensory panel and helping ensure a successful data collection. Morten Münchow would like to thank John Thompson (Coffee Nexus) and Filip Åkerblom (Scandinavian Coffee Consulting) for their invaluable inputs to his understanding of coffee roasting and coffee roasting education during their many years of work with SCAE's education system.

Conflicts of Interest: The authors declare no conflict of interest.

\section{References}

1. Nair, K.P. The Agronomy and Economy of Important Tree Crops of the Developing World; Elsevier: Amsterdam, The Netherlands, 2010.

2. Ponte, S. The 'latte revolution'? Regulation, markets and consumption in the global coffee chain. World Dev. 2002, 30, 1099-1122. [CrossRef]

3. Chambers IV, E.; Sanchez, K.; Phan, U.X.; Miller, R.; Civille, G.V.; Di Donfrancesco, B. Development of a "living" lexicon for descriptive sensory analysis of brewed coffee. J. Sens. Stud. 2016, 31, 465-480. [CrossRef]

4. Hayakawa, F.; Kazami, Y.; Wakayama, H.; Oboshi, R.; Tanaka, H.; Maeda, G.; Hoshino, C.; Iwawaki, H.; Miyabayashi, T. Sensory lexicon of brewed coffee for Japanese consumers, untrained coffee professionals and trained coffee tasters. J. Sens. Stud. 2010, 25, 917-939. [CrossRef]

5. Bhumiratana, N.; Adhikari, K.; Chambers, E. Evolution of sensory aroma attributes from coffee beans to brewed coffee. LWT-Food Sci. Technol. 2011, 44, 2185-2192. [CrossRef]

6. Buffo, R.A.; Cardelli-Freire, C. Coffee flavour: An overview. Flavour Fragr. J. 2004, 19, 99-104. [CrossRef]

7. Poisson, L.; Blank, I.; Dunkel, A.; Hofmann, T. The Chemistry of Roasting-Decoding Flavor Formation. In The Craft and Science of Coffee; Elsevier: Amsterdam, The Netherlands, 2017; pp. 273-309.

8. Giacalone, D.; Fosgaard, T.R.; Steen, I.; Münchow, M. Quality does not sell itself: Divergence between objective product quality and preference for coffee in naïve consumers. Br. Food J. 2016, 118, 2462-2474. [CrossRef]

9. Cheng, B.; Furtado, A.; Smyth, H.E.; Henry, R.J. Influence of genotype and environment on coffee quality. Trends Food Sci. Technol. 2016, 57, 20-30. [CrossRef]

10. Illy, A.; Viani, R. Espresso Coffee: The Science of Quality; Elsevier Academic Press: San Diego, CA, USA, 2005.

11. Heo, J.; Choi, K.S.; Wang, S.; Adhikari, K.; Lee, J. Cold Brew Coffee: Consumer Acceptability and Characterization Using the Check-All-That-Apply (CATA) Method. Foods 2019, 8, 344. [CrossRef]

12. Belitz, H.D.; Grosch, W.; Schieberle, P. Coffee, tea, cocoa. In Food Chemistry; Springer: Berlin/Heidelberg, Germany, 2009; pp. 938-970.

13. Clarke, R.; Vitzthum, O. Coffee: Recent Developments; John Wiley \& Sons: Oxford, UK, 2008. 
14. Schenker, S.; Handschin, S.; Frey, B.; Perren, R.; Escher, F. Pore structure of coffee beans affected by roasting conditions. J. Food Sci. 2000, 65, 452-457. [CrossRef]

15. Hofmann, T.; Frank, O.; Blumberg, S.; Kunert, C.; Zehentbauer, G. Molecular insights into the chemistry producing harsh bitter taste compounds of strongly roasted coffee. In Recent Highlights in Flavor Chemistry and Biology; Deutsche Forschungsanstalt für Lebensmittelchemie: Reising, Germany, 2008; pp. 154-159.

16. Purdon, M.P.; McCamey, D.A. Use of a 5-caffeoylquinic acid/caffeine ratio to monitor the coffee roasting process. J. Food Sci. 1987, 52, 1680-1683. [CrossRef]

17. Yeretzian, C.; Jordan, A.; Badoud, R.; Lindinger, W. From the green bean to the cup of coffee: Investigating coffee roasting by on-line monitoring of volatiles. Eur. Food Res. Technol. 2002, 214, 92-104. [CrossRef]

18. Schenker, S.; Heinemann, C.; Huber, M.; Pompizzi, R.; Perren, R.; Escher, R. Impact of roasting conditions on the formation of aroma compounds in coffee beans. J. Food Sci. 2002, 67, 60-66. [CrossRef]

19. Bicho, N.C.; Leitão, A.E.; Ramalho, J.C.; de Alvarenga, N.B.; Lidon, F.C. Impact of roasting time on the sensory profile of Arabica and Robusta coffee. Ecol. Food Nutr. 2013, 52, 163-177. [CrossRef] [PubMed]

20. Baggenstoss, J.; Poisson, L.; Kaegi, R.; Perren, R.; Escher, F. Coffee roasting and aroma formation: Application of different time- temperature conditions. J. Agric. Food Chem. 2008, 56, 5836-5846. [CrossRef] [PubMed]

21. Blumberg, S.; Frank, O.; Hofmann, T. Quantitative studies on the influence of the bean roasting parameters and hot water percolation on the concentrations of bitter compounds in coffee brew. J. Agric. Food Chem. 2010, 58, 3720-3728. [CrossRef]

22. Lyman, D.J.; Benck, R.; Dell, S.; Merle, S.; Murray-Wijelath, J. FTIR-ATR analysis of brewed coffee: Effect of roasting conditions. J. Agric. Food Chem. 2003, 51, 3268-3272. [CrossRef]

23. Wang, X.; Lim, L.T. A kinetics and modeling study of coffee roasting under isothermal conditions. Food Bioprocess Technol. 2014, 7, 621-632. [CrossRef]

24. Schenker, S.; Rothgeb, T. The roast-Creating the Beans' signature. In The Craft and Science of Coffee; Elsevier: Amsterdam, The Netherlands, 2017; pp. 245-271.

25. Maier, H. Zur Zusammensetzung kurzeitgerösteter Kaffees. Lebensm.-Chem. Gerichtl. Chem 1985, 35 , 25-33.

26. Giacalone, D.; Degn, T.K.; Yang, N.; Liu, C.; Fisk, I.; Münchow, M. Common roasting defects in coffee: Aroma composition, sensory characterization and consumer perception. Food Qual. Prefer. 2019, 71, 463-474. [CrossRef]

27. Yang, N.; Liu, C.; Liu, X.; Degn, T.K.; Munchow, M.; Fisk, I. Determination of volatile marker compounds of common coffee roast defects. Food Chem. 2016, 211, 206-214. [CrossRef]

28. Roast. Book of Roast the Craft of Coffee Roasting from Bean to Business; Roast Magazine: Portland, OR, USA, 2017.

29. Folmer, B. The Craft and Science of Coffee; Academic Press: Amsterdam, The Netherlands, 2017.

30. Kim, K.J.; Park, S.K. Changes in major chemical constituents of green coffee beans during the roasting. Korean J. Food Sci. Technol. 2006, 38, 153-158.

31. Dmowski, P.; Dabrowska, J. Comparative study of sensory properties and color in different coffee samples depending on the degree of roasting. Zesz. Nauk. Akad. Morskiej W Gdyni 2014, 84, 28-36.

32. Feria-Morales, A.M. Examining the case of green coffee to illustrate the limitations of grading systems/expert tasters in sensory evaluation for quality control. Food Qual. Prefer. 2002, 13, 355-367. [CrossRef]

33. Lawless, H.T.; Heymann, H. Sensory Evaluation of Food: Principles and Practices; Springer Science \& Business Media: New York, NY, USA, 2010.

34. Steen, I.; Waehrens, S.S.; Petersen, M.A.; Münchow, M.; Bredie, W.L. Influence of serving temperature on flavour perception and release of Bourbon Caturra coffee. Food Chem. 2017, 219, 61-68. [CrossRef] [PubMed]

35. Di Donfrancesco, B.; Gutierrez Guzman, N.; Chambers, E. Comparison of results from cupping and descriptive sensory analysis of Colombian brewed coffee. J. Sens. Stud. 2014, 29, 301-311. [CrossRef]

36. SCAA. SCAA Protocols-Cupping for Specialty Coffee; Specialty Coffee Association of America and Europe: Chelmsford, UK, 2018.

37. R Core Team. R: A Language and Environment for Statistical Computing; R Foundation for Statistical Computing: Vienna, Austria, 2014. 
38. Nebesny, E.; Budryn, G. Evaluation of sensory attributes of coffee brews from robusta coffee roasted under different conditions. Eur. Food Res. Technol. 2006, 224, 159-165. [CrossRef]

39. Giacalone, D.; Steen, I.; Alstrup, J.; Münchow, M. Inter-rater reliability of 'clean cup' scores by coffee experts. J. Sens. Stud. 2020, under review.

40. Chapko, M.J.; Seo, H.S. Characterizing product temperature-dependent sensory perception of brewed coffee beverages: Descriptive sensory analysis. Food Res. Int. 2019, 121, 612-621. [CrossRef]

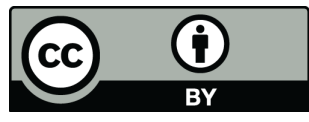

(C) 2020 by the authors. Licensee MDPI, Basel, Switzerland. This article is an open access article distributed under the terms and conditions of the Creative Commons Attribution (CC BY) license (http:/ / creativecommons.org/licenses/by/4.0/). 